Para enlazar con este artículo / To link to this article:

http://dx.doi.org/10.14198/fem.2018.32.05

Para citar este artículo / To cite this article:

Cordero Ampuero, Ángel y Esteban Maluenda, Ana. «Christine Dalnoky. El ritmo pausado del paisaje». En Feminismo/s, 32 (diciembre 2018): 135-155. Dosier monográfico: MAS-MES: Mujeres, Arquitectura y Sostenibilidad - Medioambiental, Económica y Social, coord. María-Elia Gutiérrez-Mozo, DOI: 10.14198/fem.2018.32.05

\title{
CHRISTINE DALNOKY. EL RITMO PAUSADO DEL PAISAJE
}

\author{
CHRISTINE DALNOKY. \\ THE SLOW RHYTHM OF LANDSCAPE
}

\author{
Ángel Cordero AmPUERO \\ Universidad Politécnica de Madrid \\ Escuela Técnica Superior de Arquitectura de Madrid \\ angel.cordero@upm.es \\ https://orcid.org/0000-0002-1927-7304 \\ Ana Esteban MalUENDA \\ Universidad Politécnica de Madrid \\ Escuela Técnica Superior de Arquitectura de Madrid \\ ana.esteban.maluenda@upm.es \\ http://orcid.org/0000-0002-0482-8214
}

\section{Resumen}

Este trabajo presenta un recorrido por la obra de Christine Dalnoky, referencia clave para el paisajismo contemporáneo en las últimas décadas. Alineada con la corriente crítica que plantea la práctica de la arquitectura del paisaje como recuperación de la memoria del lugar -en contraposición a las teorías arquitectónicas enfocadas al programa-, se expone su análisis como intersección de planteamientos ideológicos, dialéctica espacial y prevalencia de los ritmos, en coherencia con su evolución personal. La biografía de Christine Dalnoky se perfila en dos etapas: la primera en París, marcada por su asociación a Michel Desvignes desde 1988; la segunda en una pequeña localidad de Provenza, donde en 2002 fundó su nuevo estudio junto a Patrick Solvet. Este drástico alejamiento de la metrópolis se presenta en paralelo al desarrollo de sus proyectos, en especial el del Parque del Agua en Zaragoza, donde el ritmo pausado del paisaje se impone definitivamente a la agitación urbana. (CC BY 4.0)

Feminismo/s 32, diciembre 2018, pp. 135-155 
Palabras clave: paisajismo, arquitectura, urbanismo, diseño urbano, paisaje.

\begin{abstract}
This paper is displayed like a journey across the work of Christine Dalnoky, key referent for contemporary landscape architecture. Taking part of the critical movement towards a new landscape architecture focused on memory recovering of the site -in contrast with architectonical theories focused on program-, the analysis is presented as a crossing between ideological positions, spatial dialectic and prevalence or rhythms, in coherence with her personal evolution. Christine Dalnoky biography is divided in two periods: first settled in Paris and identified with her association from 1988 with Michel Desvignes; second moved to a little town in Provence, where she founded a new practices with Patrick Solvet. This radical removal from metropolis is shown in parallel with her projects development, in special with this of Parque del Agua in Zaragoza, where slow rhythm of landscape is imposed definitely over urban agitation.
\end{abstract}

Keywords: Landscape Architecture, Architecture, Urbanism, Urban Design, Landscape.

«Un paisaje es un espacio creado deliberadamente para acelerar o frenar los procesos naturales» (Jackson 8) ${ }^{1}$.

Christine Dalnoky es una de las figuras pioneras del nuevo paisajismo y, por su rebeldía, se ha convertido en un modelo de coherencia vital respecto a sus planteamientos profesionales. Parafraseando el nuevo concepto de «suburbanismo» (Marot, Suburbanismo 12), se podría decir que la suya es una trayectoria de 'subreferencia', por oposición a los criterios impositivos que vienen siendo costumbre en los ámbitos del paisajismo, el urbanismo o la arquitectura desde, al menos, los inicios del Renacimiento (Tafuri 15-39).

Este artículo busca hacer una recopilación crítica de la trayectoria de dicha paisajista y, en alguna medida, paliar la relativa ausencia de sus trabajos en los estudios científicos del área. Es obvio que este olvido no es casual, y no deja de ser coherente con la actitud discreta, casi anónima, que se esfuerza

1. Texto original en inglés: «A landscape is a space deliberately created to speed up or slow down the process of nature» (Traducción de Ángel Cordero).

Feminismo/s 32, diciembre 2018, pp. 135-155 
por cultivar la propia Christine Dalnoky; sin embargo, en este esfuerzo se pierde la oportunidad de sumar al debate intelectual, en su auténtica dimensión propositiva, las ideas pioneras de su creatividad y su intensidad ética.

\section{ITALIA}

La recuperación del paisajismo en la Francia de la década de 1980, como revulsivo frente a décadas de abandono de la disciplina en aras de la zonificación urbana, la planificación estratégica del territorio y sus infraestructuras, tuvo como detonante paradójico la puesta en marcha de las villes nouvelles (equivalentes a las británicas new towns), que requerían el apoyo de esta disciplina como 'paliativo' a la urbanización moderna (Marot, The Reclaiming of Sites 47). En 1976 se creó la Ècole Nationale Supérieure du Paysage en Versalles, donde se focalizó un auténtico think tank sobre las posibilidades y las vías de renovación de la disciplina, capitaneado por la figura de Michel Corajoud:

La visión que se elabora hoy en Versalles no se limita a un mero cambio de prioridades entre la edificación y el paisaje, sino que se fundamenta en la 'lectura y escritura' del lugar propiamente dicho. Dicha visión está menos enfocada al programa de determinado proyecto edificatorio que a la exploración de las posibilidades que ofrecen las características del lugar y su fenomenología. De este modo, subraya una aproximación crítica y reflexiva para crear nuevos paisajes. (Marot, The Reclaiming of Sites 48) ${ }^{2}$

Christine Dalnoky estudió Bellas Artes primero en París y después en la École Nationale Supérieure du Paysage de Versalles. Tras colaborar con el propio Corajoud y Alexandre Chemetoff en París, y con Renzo Piano en Génova, obtuvo la beca de la academia de Francia en Roma y residió en la Villa Medici entre 1986 y 1988. En Italia, donde ya había eclosionado su vocación paisajística, se encontró con quien sería su primer socio, Michel Desvignes, compañero de la academia francesa, de formación más científica, pero interesado, como ella, en el jardín como disciplina artística. De hecho, ambos fueron

2. Texto original en inglés: «The vision being elaborated in Versailles today is not limited to merely changing priorities from building to landscape but instead takes as its starting point the 'reading and writing' of the site itself. Such a view is less focused on the program of a proposed building project than on exploring the possibilities of site characteristics and hidden phenomena. As such, it outlines a critical and reflective approach to making new landscapes» (Traducción de Ángel Cordero).

Feminismo/s 32, diciembre 2018, pp. 135-155 
los primeros paisajistas franceses becados en Roma, desde donde pudieron reflexionar en profundidad sobre el paisaje latino como punto de encuentro entre naturaleza, agricultura, ciudad y arte. En estos territorios limítrofes, sumergidos en los entornos suburbanos renacentistas, analizaron en detalle el jardín de Bomarzo, con su atractivo surrealista incentivado por el éxito internacional de la novela (y ópera) de Manuel Mújica Láinez. Entre sus conclusiones propusieron recuperar el sentido del jardín en el paisajismo contemporáneo como paisaje simbólico, a modo de catalizador de una reflexión fundamental: la relación entre el entorno natural y el medio artificial en su crecimiento desbocado. Así, de vuelta en Francia, en 1988 nació el estudio Desvigne \& Dalnoky.

\section{PARÍS-LONDRES}

Su primer reconocimiento, el jardín parisino de la Rue de Meaux (Square de Bouleaux, 1989-1992), vino de la mano de su colaboración con el arquitecto «estrella» Renzo Piano, director del encargo y autor del edificio de viviendas sociales. Planteado como un soto de abedules en medio de la gran ciudad, los paisajistas consiguen recrear una atmósfera envolvente, que sorprende en un jardín privado de manzana, al tiempo que se ciñen a un ajustado presupuesto, dado que los árboles se habían desechado en un vivero. El éxito de este jardín, junto con la irrupción de nuevas propuestas paisajísticas más acordes a la nueva sensibilidad social ante el medio ambiente, permitió a Desvigne y Dalnoky difundir sus principios muy pronto, con todos los riesgos que conllevaba su papel pionero: la exposición a los mass media (incluidos los más profesionalizados), el consumo rápido de sus ideas en el marasmo de las tendencias e, incluso, la mixtificación con cualquier otra aproximación de interés. Desde finales de la década de 1980, las imágenes más superficiales, tan necesarias para amplificar el mensaje, se venían consumiendo con voracidad acrítica, y fue más aún en la década siguiente, cuando en todas la escuelas de arquitectura y paisaje las ideas se presentaban mezcladas con cualquier otra que connotara «verde».

Con todo, esta popularidad dio pie a nuevos encargos o concursos restringidos en los que insistieron en sus ideas clave más allá de la pequeña escala del jardín:

Feminismo/s 32, diciembre 2018, pp. 135-155 
El sprawl no es inevitable de ninguna manera, y dado que en este territorio no hay lugar para la nostalgia, dado que no hay un estadio histórico al que podamos hacer referencia, soñamos con ciudades enraizadas en su territorio, ciudades donde uno pueda sentir la pendiente de una colina, sentir la frescura de los valles, seguir la corriente del agua y el ciclo de las estaciones, ciudades donde las distancias se puedan medir, donde caiga realmente la noche, en las que el tiempo se asiente en la tierra, en la piel del paisaje. (Desvignes y Dalnoky 36$)^{3}$

Así se fueron sucediendo los proyectos a media y gran escala, como la propuesta de concurso para el viaducto de Millau (1994), donde no sólo proyectaron un sistema de paisaje cambiante, sino que también aprovecharon para investigar sobre cómo cartografiar la sucesión espacio-temporal del paisaje existente y la complejidad de los impactos en su intervención. Una exploración gráfica que se sucedió en los proyectos vinculados al ferrocarril de alta velocidad TGV, donde dicha infraestructura, escasamente sensible al territorio, incorporó a los profesionales del paisaje para responder a una demanda social más respetuosa con un discurso contemporáneo. De nuevo, Dalnoky y Desvignes se preguntaban cómo el entorno, con su ritmo centenario, podía registrar sistemas temporales tan acelerados: de esa manera, la representación de los «patrones» del paisaje agrario se convirtió en leitmotiv para los proyectos paisajísticos de las estaciones de Avignon o Marsella.

A través de sus propuestas en los años 1990, Desvignes \& Dalnoky afrontaron uno de los retos fundamentales del paisajismo contemporáneo:

una toma de conciencia orientada, por un lado, a considerar los espacios públicos (proyectos urbanos) como paisajes y, por otro, a ver los paisajes (extensiones rurales) como espacios públicos y por tanto como posibles sujetos de proyectos. (Marot, The Reclaiming of Sites 53) ${ }^{4}$

3. Texto traducido del inglés: «Urban sprawl is in no sense inevitable and, since there is no nostalgia here, since there is no earlier state to which we can make reference, we dream of cities rooted to their landscape, cities where one can feel the slope of a hill, sense the freshness of valleys, follow the flow of water and the cycle of the seasons, cities in which distances can be measured, in which night truly falls, in which time is inscribed on the earth, on the skin of the landscape» (Traducción de Ángel Cordero).

4. Texto original en inglés: «an awareness that is directed, on the one hand, to consider public spaces (urban projects) as landscapes and, on the other, to see landscapes (rural expanses) as public spaces and therefore as possible objectives of projects» (Traducción de Ángel Cordero).

Feminismo/s 32, diciembre 2018, pp. 135-155 
Así, alineados con las tesis de Sébastien Marot, junto a Michel Corajud y otros paisajistas de la École Nationale Supérieure du Paysage de Versalles, Desvignes y Dalnoky fueron dando cuerpo a esa nueva disciplina del «suburbanismo», acuñada por el propio Marot a través de un manifiesto ${ }^{5}$ como alternativa necesaria para la arquitectura del paisaje, que se basaba en cuatro planteamientos:

la memoria, o anamnesis, de las cualidades del emplazamiento; la visión del emplazamiento y del proyecto como procesos más que como productos; la lectura en espesor, y no solamente en planta, de los espacios abiertos; y, finalmente, el pensamiento relativo: una concepción del emplazamiento y del proyecto como campos de relaciones, más que como disposiciones de objetos. (Marot, Suburbanismo 10)

A finales del siglo XX, su colaboración en el proyecto de Richard Rogers and Partners para la península de Greenwich, antesala de la fastuosa Millennium Dome, les acabó de consagrar como actores de la gran representación global del diseño. Su propuesta, que consistía en una gran repoblación forestal como respuesta a la insalubridad del suelo, volvía a expresar la intención de reencontrar las claves agrarias de un paisaje perdido: el de las arboledas de ribera y sus estrictas leyes de plantación. Junto al planteamiento ideológico, se yuxtaponían dos sensibilidades ancladas a la memoria del paisaje, símbolos de la recuperación del territorio humanizado, pero no expoliado, de la «naturaleza intermedia». En primer lugar, la sensibilidad del espacio que se abre entre la trama ortogonal y se ondula con indolencia en un recorrido como el de la Caperucita que se entretiene, absorta, en la contemplación de los troncos alineados y las hojas brillantes; y, en segundo lugar, la sensibilidad del tiempo del crecimiento, bosque y sotobosque que avanzan lentamente y colonizan poco a poco la ribera, desde los tiernos plantones a los ejemplares maduros, y vuelta a empezar:

5. En sus palabras, más bien como un anti-manifiesto frente al «sobreurbanismo»o «superurbanismo», «tendencia mayoritaria, bastante fácil de identificar, de la arquitectura contemporánea teorizada por Rem Koolhaas 'a través de un «manifiesto retroactivo' compuesto en honor y gloria de una metrópoli americana» (Marot, Suburbanismo 13).

Feminismo/s 32, diciembre 2018, pp. 135-155 
Un paisaje está completamente vinculado a los efectos de la naturaleza y el tiempo: el proceso hidrológico, la climatología y la sucesión; y la alternancia del día y la noche, el sol y la luna. (Marot, The Reclaiming of Sites 51) ${ }^{6}$

\section{ZARAGOZA}

Terminada su asociación con Michel Desvignes, en el año 2002 Christine Dalnoky fundó l'Atelier de Paysage. Se alejó entonces de París y se estableció en el espectacular pueblo de Gordes, en el departamento de Vaucluse (en el parque del Luberon), donde en 2004 escribió con Patrick Solvet, su nuevo socio, el libro Tous Ego. L'abecedaire. Ajena al proyecto espacial, su primera obra en este retiro provenzal fue un ensayo literario donde, más allá de estrategias o ideologías, discurría por una suerte de deriva ${ }^{7}$ crítica, provocadora y abiertamente contraria a cualquier sistema determinista en la cultura, el arte o el diseño.

Dado el éxito alcanzado por sus proyectos en la década anterior, así como las relaciones con algunas de las principales firmas internacionales de arquitectura, en ese momento los cambios personales y profesionales fueron drásticos. Incluso, al apartarse de la metrópoli, su traslado geográfico reflejaba una coherencia relevante respecto a la recuperación de la memoria (su región de procedencia ${ }^{8}$ no estaba lejos de la Alta Provenza donde se había instalado) del paisaje rural, más allá de la sutil huida de los resortes impositivos del poder de los medios. Desde entonces, también ha venido asumiendo las consecuencias de este peculiar distanciamiento: al alejarse de la contaminación de las tendencias, el alcance profesional de sus propuestas disminuyó y, con ello, su capacidad de incidir en los planteamientos críticos del paisajismo contemporáneo.

6. Texto original en inglés: «a landscape is fully bound into the effects of nature and time: process of hydrology, weathering, and succession; and the alternation of day and night, sun and moon» (Traducción de Ángel Cordero).

7. El término es explícitamente situacionista, en un sistema literario donde no cabe el término recorrido (ni tan siquiera narrativa), dado su carácter errático, pero donde la subestructura es marcadamente ordenada, definida y precisa. Hasta cierto punto, y con permiso de Michel Desvignes, se podría decir que Tous Ego reproduce en términos narrativos la lógica paisajística del proyecto de la península de Greenwich.

8. En una reciente publicación de sus redes sociales identificaba el paisaje rural de Ceyzériat (Bourg-en-Bresse, departamento de Ain) como «el campanario de mi infancia».

Feminismo/s 32, diciembre 2018, pp. 135-155 
Por otra parte, su discurso ganó independencia y pudo añadir a sus ideas previas la reivindicación explícita de recuperar el placer de la naturaleza en la ciudad (Alba): un concepto habitual, pero a menudo obviado por la arquitectura o el paisajismo, tal vez por sus connotaciones frívolas o simplemente ingenuas, incluso en el mejor sentido de la palabra: «[...] nuestra tarea era no perder jamás la ambición poética de este parque, que debe convertirse para los usuarios en un lugar de emociones, placer y dulzura» (Dalnoky 50).

Estas palabras se refieren al proyecto más difundido de l'Atelier de Paysage: el Parque del Agua Luis Buñuel en Zaragoza. Proyectado junto a los arquitectos Margarita Jover e Iñaki Alday como parte del recinto de la Exposición Internacional de 2008, dedicada al agua, el proyecto venció en un concurso de ideas convocado en enero de 2005, cuyo jurado presidió Michel Corajoud, debido a:

[...] la sensibilidad de la propuesta y por la actitud cultural adoptada para el diseño del parque. Por la atención a aspectos como la materialización del agua en el parque, la materialización del suelo y la materialización de los sistemas constructivos. Una propuesta de gran coherencia global, con las premisas de partida del concurso, y con una formalización muy satisfactoria, especialmente en algunos enclaves, como el nuevo frente hacia la avenida de Ranillas, con un canal-depósito, elevado en algunos tramos, que da apoyo a las nuevas edificaciones proyectadas. (De la Cal 71-72)

Dentro de la indudable coherencia del proyecto, no deja de sorprender la distancia entre el discurso de la paisajista y su colega arquitecta:

El proyecto ya no habla de belleza, sino de vida;

ya no de estética, sino de ética;

ya no de moda, sino de permanencia y roce. (Jover 15)

No obstante, la arquitecta compartía implícitamente con Dalnoky la idea de que el proyecto «es la planificación de una transformación cuyo planteamiento parte de un posicionamiento cultural respecto la realidad que nos rodea» (Jover 13). Éste es un hecho explícito en la Zaragoza que se abre al entorno natural, para asumir que el parque no es un mero diseño urbano, sino un lugar de encuentro entre la extrema domesticación de los nuevos ensanches y las orillas salvajes del caudaloso río Ebro. Un planteamiento

Feminismo/s 32, diciembre 2018, pp. 135-155 
ideológico ${ }^{9}$ que, a finales de los años 1990, se integraba tanto en la teoría del «suburbanismo» de Marot como en sus predicciones sobre el papel determinante de esta nueva forma de entender la profesión de arquitecto del paisaje. En este sentido es donde la propuesta del parque del agua parece más pionera, más radical en su acepción etimológica y, por tanto, más difícil de digerir por nuestra sociedad de la comunicación. En pleno siglo XXI, con una sociedad consciente de su compromiso medioambiental y de sus límites planetarios, la ciudad de Zaragoza no podía permitirse robar al río el meandro de Ranillas, a no ser que lo hiciese imitando, también en sus riesgos, a aquellos agricultores que aprovechaban y temían las crecidas del río. Así, gracias a esta intervención, el barrio ACTUR se situó mirando al cauce con admiración y odio: con el tiempo una hilera de equipamientos un tanto desordenados parece intentar cerrar el parque, en un diseño urbano que se arriesga a metamorfosearse en mall y un canal de depósito, paralelo a la Avenida de Ranillas (auténtica domesticación del Ebro), que podría acabar siendo un estanque inocuo, con patitos para decorar el paseo comercial (Fig. 1).

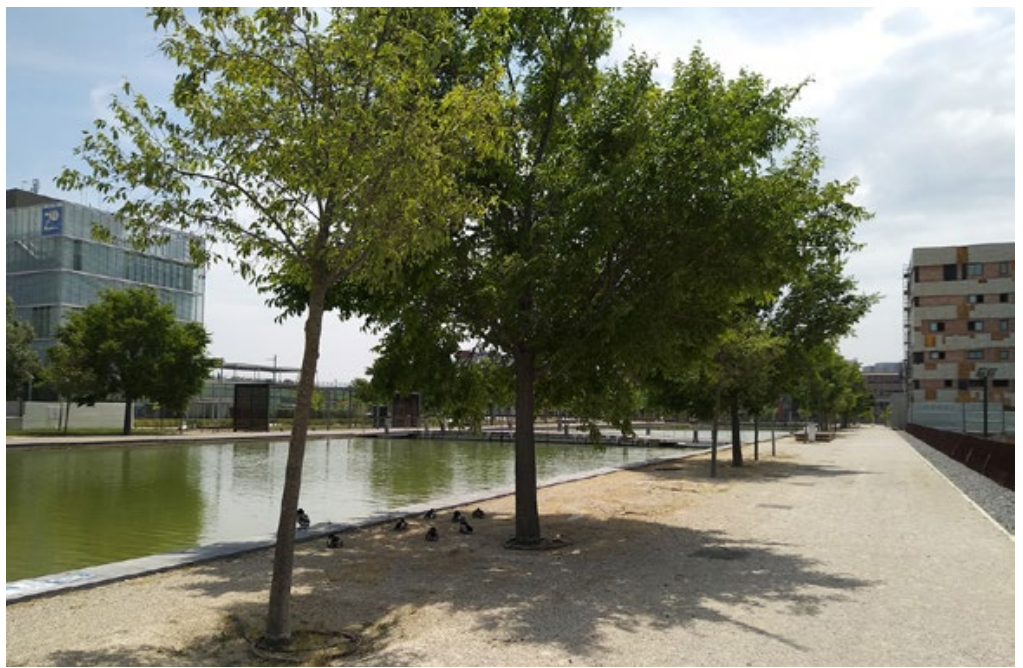

Figura 1.

9. Aunque Margarita Jover intente evitar el término «ideológico» y sustituirlo por el de «cultural».

Feminismo/s 32, diciembre 2018, pp. 135-155 


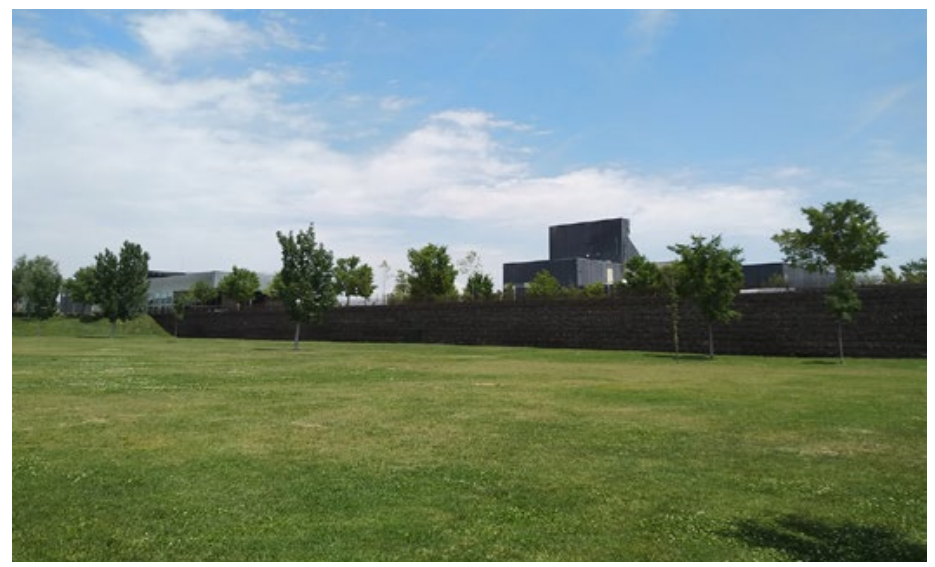

Figura 2.

Sin embargo, bastaba con cruzar alguna de las primeras praderas en depresión, o con asomarse a la mota norte (el paseo sobre el dique), para volver a confiar en la capacidad de la naturaleza de imponer algunas de sus leyes y volver a disfrutarlas: la humedad extrema del suelo de la gran pradera (Fig. 2), al límite de la capa freática, bastaba para sentir la intensidad del acuífero; el paseo elevado se entendía como refugio atávico del cauce principal (Fig. 3);

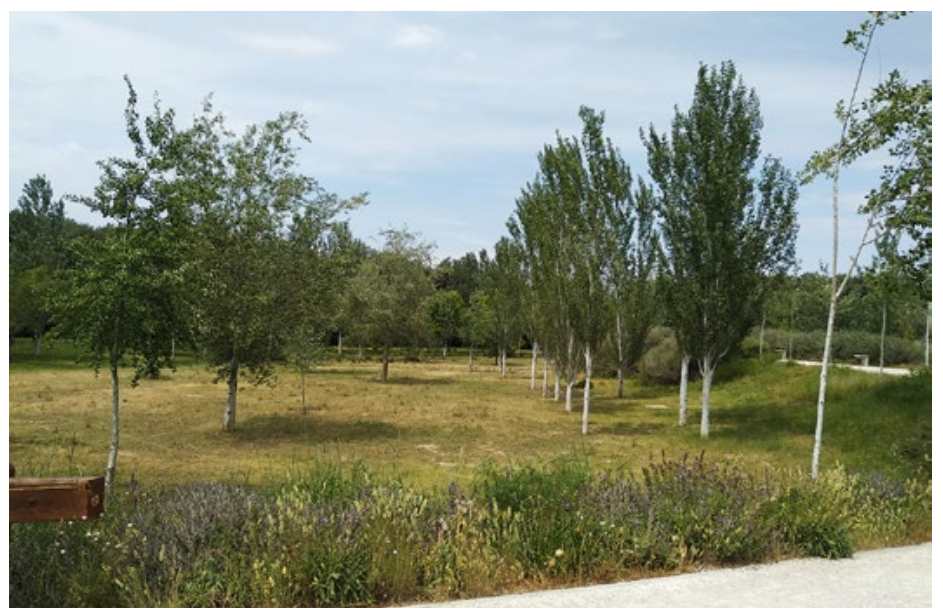

Figura 3.

Feminismo/s 32, diciembre 2018, pp. 135-155 


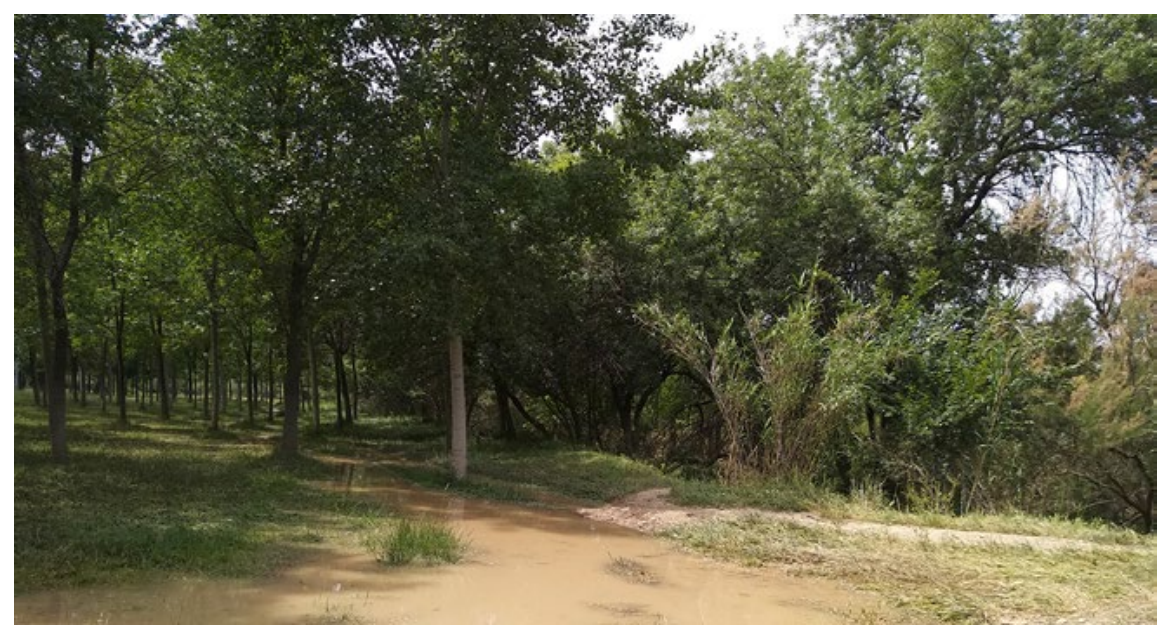

Figura 4.

o, finalmente, uno podía sentir la llamada del soto salvaje en la ribera frondosa del bosque plateado reivindicado poéticamente por el proyecto y que hoy disfrutan los corredores, los ciclistas y los paseantes más aventureros (Fig. 4). De nuevo, las palabras de la paisajista ilustran su intención: «¡Nuestra ambición será anclar el proyecto a la historia del suelo para que se integre más fácilmente, comprender antes para poder soñar después!» (Dalnoky 49).

Tal vez el aspecto más decepcionante de la situación actual del parque sea su fracaso como gran infraestructura hidráulica, demasiado compleja (López y Garín 138-149), demasiado costosa, demasiado pionera o, simplemente, demasiado poco rentable. Esta situación priva al visitante del disfrute de la tecnología que se había proyectado, sostenible y transparente: en la actualidad, a falta de una mayor exigencia social, el acueducto de tratamiento ha dejado de funcionar y se asemeja a una ruina contemporánea (Fig. 5). Al menos, el sistema del agua se recupera en los humedales de tratamiento, donde de nuevo asoma como un medio natural domesticado, si no capaz de completar la limpieza del agua, al menos capaz de dar cobijo a un ecosistema prácticamente salvaje, apenas dominado en las alturas desde la Torre del Agua (Fig. 6).

Feminismo/s 32, diciembre 2018, pp. 135-155 
Ángel Cordero Ampuero y ANA Esteban Maluenda

Christine Dalnoky. El ritmo pausado del paisaje

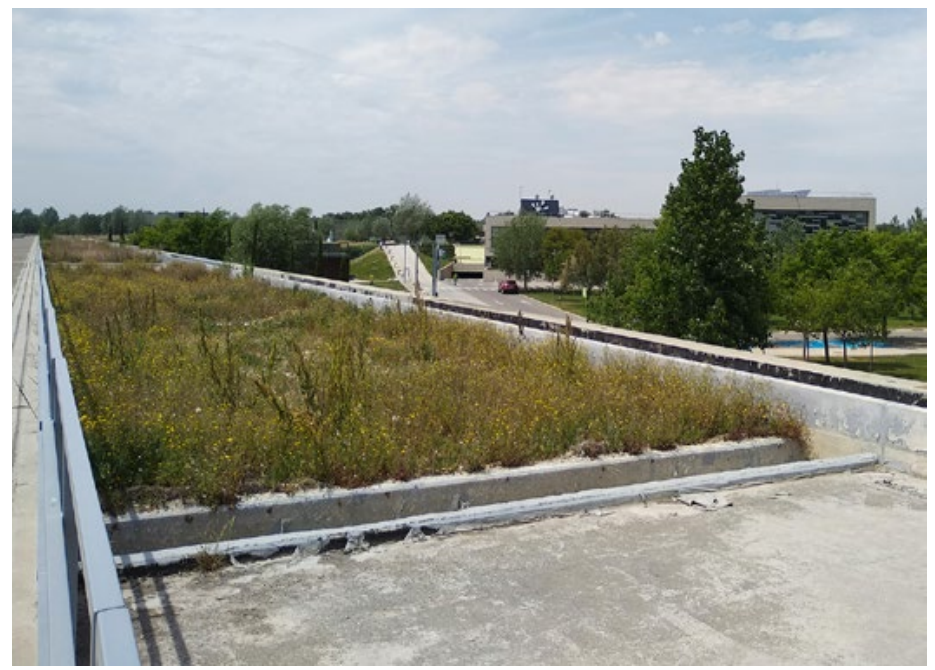

Figura 5.

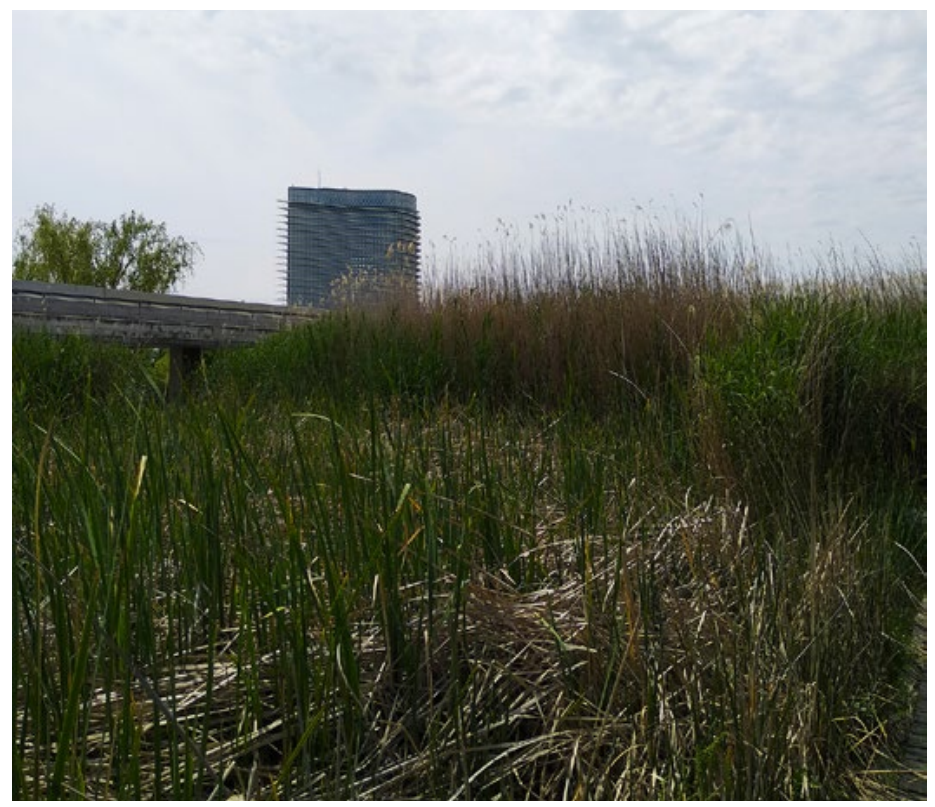

Figura 6.

Feminismo/s 32, diciembre 2018, pp. 135-155 


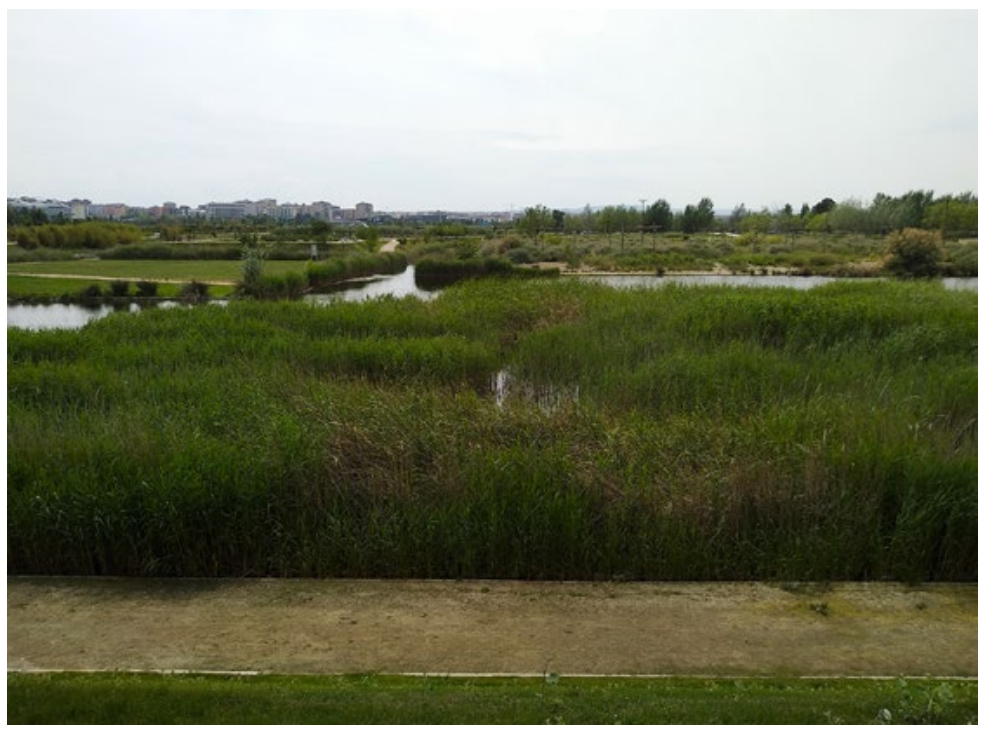

Figura 7.

En la estructura paisajística del parque, la lógica del agua (asimilada a la naturaleza, al menos desde los humedales de tratamiento) dialoga con la lógica agrícola (como última traza humanizada memorable). Así, desde la línea del azud (Fig. 7), el auténtico parque paisajístico se extiende por la mayor parte del antiguo meandro y deja su borde sudoriental a expensas del «aprovechamiento urbanístico»: el canal de aguas bravas que rodeaba la Torre del Agua, la plaza sur y los restos de arboretum que aún resisten el embate de los usos lucrativos (Fig. 8). Hacia el noroeste, las edificaciones o sus ruinas, los jardines privatizados o los usos compatibles se integran en el dominio de las aguas (Fig. 9), entre los canales y las balsas. A salvo, los paseos elevados, donde los ciudadanos ya se han vuelto visitantes, voyeurs de un territorio «que fabrica el paisaje, claramente identificativo y patrimonial» (Dalnoky 50), que no es de su propiedad ${ }^{10}$, pero que se les permite disfrutar (Fig. 10):

10. Sin duda no estarán de acuerdo los propietarios de los negocios situados en los recintos privados del parque, como tampoco algunos de sus clientes.

Feminismo/s 32, diciembre 2018, pp. 135-155 
Ángel Cordero Ampuero y ANA Esteban Maluenda

Christine Dalnoky. El ritmo pausado del paisaje

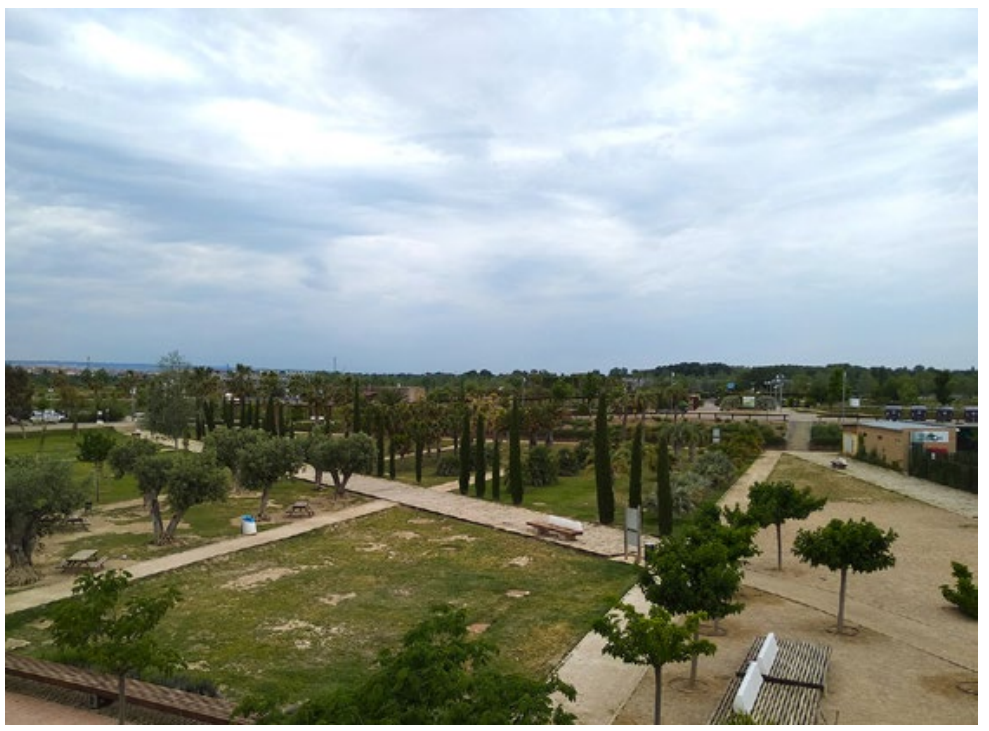

Figura 8.

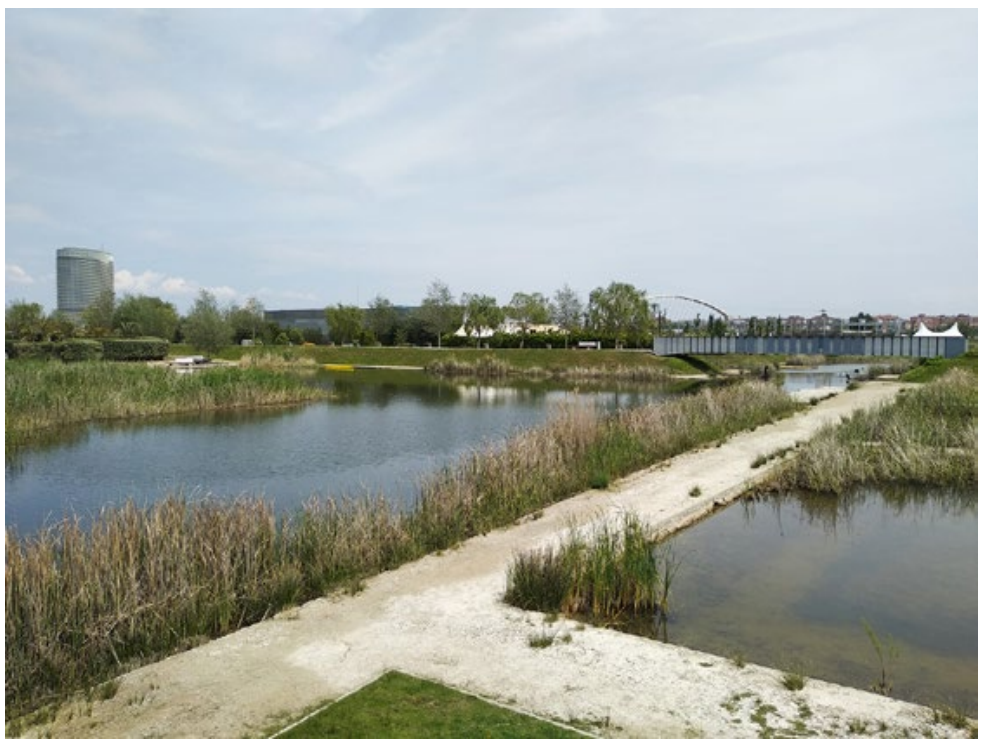

Figura 8.

Feminismo/s 32, diciembre 2018, pp. 135-155 


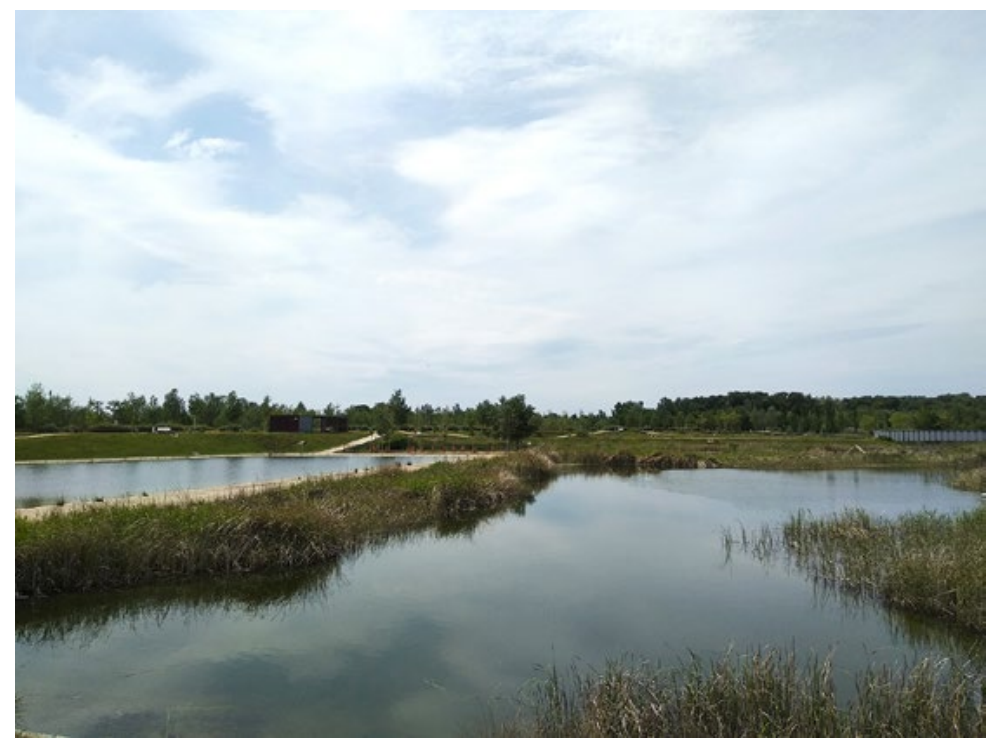

Figura 10.

Este suelo trabajado y fecundo es un bien muy preciado: su uso cambia y, por lo tanto, modificaremos la textura de los campos para adaptarla a nuevas prácticas y formas de gestión. Este corazón abierto aparece como un gran claro en el bosque plateado. (Dalnoky 52-53)

Sin embargo, el choque de actitudes resultaría inevitable. Como en todo conflicto, el tiempo acabaría mediando en los ritmos del paisaje y, por acto reflejo, en el de todo paisajismo sensible. Visto con perspectiva, hoy algunas paradojas asombran. Por ejemplo, mientras que en el embarcadero asoman unos desproporcionados patines en forma de cisne, en las balsas del extremo sudoeste ha anidado alguna familia de cisnes auténticos, lo que representa el lado más hilarante de la dialéctica entre artificio y naturaleza (Fig. 11):

Las decenas de miles de vegetales que hemos plantado crecerán y se desarrollarán, los horizontes se modificarán, las estaciones, la luz, la temperatura y los usos transformarán este lugar, haciéndolo más diverso y vivo. Este parque es un paisaje del Ebro que concentra toda la historia de su territorio para reproducir las emociones que suscita. Es, por tanto, un jardín, un inmenso jardín lúdico, sensible y sensual, un manifiesto humano. (Dalnoky 55)

Feminismo/s 32, diciembre 2018, pp. 135-155 


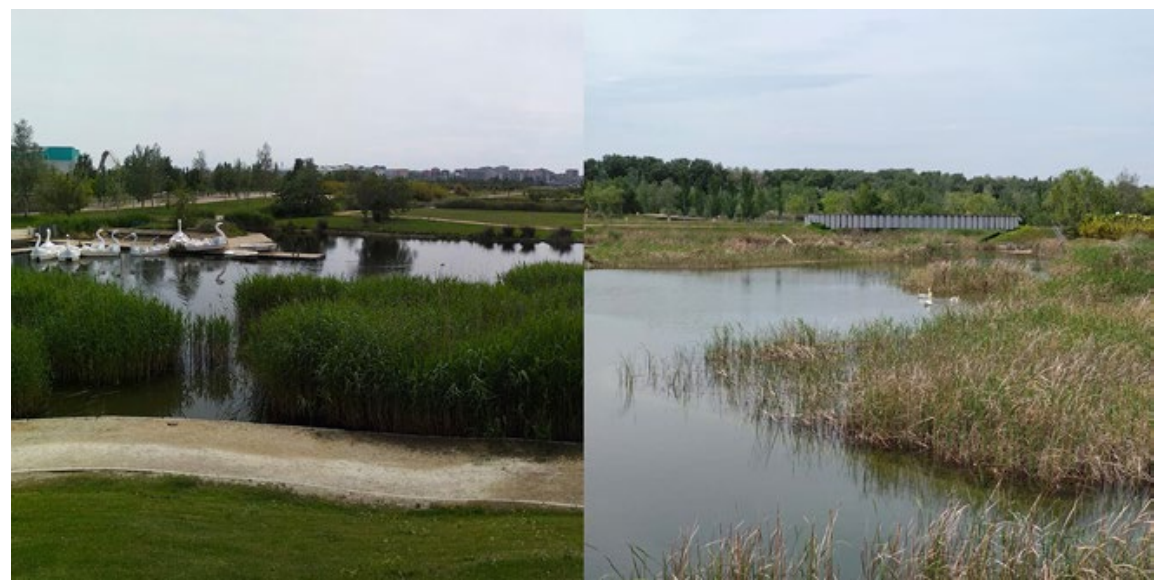

Figura 11.

Esta es, finalmente, la gran lección de Zaragoza. Su parque Luis Buñuel es un ejemplo pionero de paisaje abierto a la ciudad, de territorio debidamente conjurado; pero son y serán los ritmos de ese paisaje -los días y las noches, el devenir de las estaciones, las crecidas del río, las inundaciones y las sequías o el paso de los años- y los recuerdos de sus gentes, los que harán de este paisaje un parque ligado a la experiencia urbana. En definitiva, esos ritmos pausados que sólo pueden proyectarse con la generosidad de quien sabe que su obra sólo se completará a lo largo de generaciones, cuando los niños que hoy disfrutan de este paisaje del Ebro puedan rememorar con sus nietos la antigua experiencia del meandro.

\section{PROVENZA}

Entre las decisiones vitales de Christine Dalnoky destaca la búsqueda del ritmo del paisaje, que ella encuentra mejor desde la perspectiva rural de la Provenza interior -a pesar de su deterioro irrevocable- que desde las metrópolis inclementes, donde los pulsos se miden en nanosegundos de información. El conflicto entre la destrucción del territorio y la recreación del paisaje se ha identificado como la batalla perdida de finales del siglo XX: 
cada paisajista francés trabaja al año en un área correspondiente a la ochentava parte de la tierra modificada por los incendios forestales, o a una milésima parte de la superficie que se urbaniza. Trabajando con estas cifras, llevaría 2700 años simplemente rediseñar el paisaje agrícola que actualmente ha dejado de serlo. (Schröder 86) ${ }^{11}$

En contraste, la última década, cuando la crisis económica hubo paliado este deterioro, parece marcada por otra batalla perdida: la del tiempo. Éste es un conflicto paralelo y correlativo al que enfrenta el desarrollo urbano con su -aparentemente- antagónico medio natural, puesto que sus ritmos son los que ahora ponen en peligro nuestra propia subsistencia. Si el ritmo de los medios de transporte y, más tarde, de su consecuente explotación de los recursos decidían las crisis ecológicas del siglo XX, hoy se les ha sumado la ansiedad de la información, incluso la que se da en el propio consumo ideológico:

Mientras los pueblos han salido de la agenda, el discurso urbano-céntrico ha llegado a ser una especie de profecía autocumplida en la que las grandes ciudades son mayores, más interesantes y mejores, mientras las pequeñas ciudades y los pueblos se quedan más o menos abandonados a su suerte ${ }^{12}$. (Carlow 6)

Por tanto, y parafraseando a Dalnoky, la ciudad necesita abrirse al menos una vez más al ritmo del paisaje como recurso higiénico, representado en el medio rural (Beveridge y Rocheleau 45-49). Y, de nuevo, como entre aquellos paisajistas pioneros del siglo XIX y sus epígonos del XX,

En cierto sentido somos como un pintor que tuviera un solo bote de pintura para pintar un edificio de diez pisos. Bajo tales circunstancias sería frustrante

11. Texto original en inglés: «every French landscape architect Works on an area per year corresponding to an eightieth of the land changed by forest fires, or a thousandth of the area that became urbanized. Working on these figures, it would take 2700 years merely to redesign the landscape of all the agricultural land that has currently been set aside» (Traducción de Ángel Cordero).

12. Texto original en inglés: «Where villages and small towns have not been in the agenda, the city-centred discourse becomes almost a self-fulfilling prophecy with cities becoming larger and better, more interesting and better, whereas villages and small towns are more or less left to their own devices» (Traducción de Ángel Cordero).

Feminismo/s 32, diciembre 2018, pp. 135-155 
y erróneo tratar de pintar el edificio entero. Así que el bote de pintura deberá usarse para otra cosa. (Schröder 86) ${ }^{13}$

Desde l'Atelier du Paysage, Christine Dalnoky no ha cesado de intervenir en proyectos con el mismo propósito. Uno de los más interesantes de los últimos años es el Plan Integral de Recualificación POI (Piano Operativo Integrato) de los espacios rurales periurbanos y los poblados de la ciudad de Foggia (Cipriani y Farnè 126-135). Realizado entre 2011 y 2012 bajo la dirección del arquitecto Efisio Pitzalis, el proyecto se compone de un masterplan y de una serie de proyectos puntuales de regeneración, como los cinturones verdes para los poblados de Segezia e Incoronata o el paisajismo de la carretera y los núcleos dispersos en el paisaje de los poblados. El área, que formó parte de las transformaciones acometidas por el gobierno fascista en la Bonifica del Tavoliere (Puglia), como parte de la política de colonización agraria, se integra así como paisaje cultivado en el territorio suburbano de la ciudad de Foggia. En este sentido, la propuesta recupera una parte importante de la memoria colectiva del Tavoliere, ligado a la puesta en riego y a la actividad agrícola, al tiempo que propone esta respuesta como alternativa a la devastación del sprawl o dispersión suburbana:

El paisaje en cambio se percibe como un medio para resistir la homogeneización del entorno mientras se ponen en valor los atributos locales y un sentimiento colectivo de lugar. (Corner 13) ${ }^{14}$

Más vinculado a su retiro provenzal, en el área de Luberon se sitúa otro de sus proyectos emblemáticos de la última década: la conversión en infraestructura turística de las minas de ocre de Bruoux (Gargas) en colaboración con la oficina de arquitectura de François Defrain y Olivier Souquet (De-So). En este caso, mientras la ideología implícita -no tanto la explícita-se mantiene, los parámetros de la intervención cambian por completo: la memoria del

13. Texto original en inglés: «In some way we are like a house-painter who has only one bucket of paint to cover a ten story building. Under such circumstances it would be hopeless and wrong to try to paint the whole building. So, the single bucket of paint has to be used for something else» (Traducción de Ángel Cordero).

14. Texto original en inglés: «Landscape is instead seen as a means to resist the homogenization of the environment while also heightening local attributes and a collective sense of place» (Traducción de Ángel Cordero).

Feminismo/s 32, diciembre 2018, pp. 135-155 
paisaje no se ancla a la actividad agrícola, sino a la minería; la intervención no vincula el territorio a los nuevos desarrollos urbanos, sino a los nuevos usos turísticos -no menos invasivos- que colonizan la naturaleza; y la escala abandona las infraestructuras territoriales y vuelve a la intimidad del jardín, del diseño urbano, como actitud simbólica de una sensibilidad alternativa. Se reclama así «un lugar, un paisaje y una cápsula del tiempo» (Defrain, Souquet y Dalnoky 24) que se vuelve a plantear en los mismos términos resistentes que caracterizaron sus primeros diseños junto a Michel Desvigne:

En particular, las intervenciones marginales pueden parecer obras de arte. La gente aprende a mirar de una forma nueva sus paisajes y la forma en que han evolucionado. Si no puedes cambiar el aspecto del paisaje en gran medida, al menos puedes influir en cómo la gente lo percibe. Un enfoque marginal en términos de paisaje y placer. (Schröder 86) ${ }^{15}$

\section{CONCLUSIÓN}

[...] porque cualquiera que sea la semilla que se plante en la tierra, seguramente no llegará a disfrutarse hasta mucho después de la muerte del jardinero. Este es el sentido. Este es el sentido de la metáfora de la continuidad que Michel Corajoud usa para contrastar sus teorías con las ideologías de la dominación y la imposición obstinada: la de una conversación. (Marot, The Reclaiming of Sites 50) ${ }^{16}$

Pese a todo, la capacidad de Christine Dalnoky para provocar reflexiones se ha visto penalizada por el alejamiento de las metrópolis del poder, de los medios de comunicación y de los influencers del panorama cultural. Paradójicamente, su máxima coherencia respecto a una disciplina que hace apenas dos décadas pretendía ser madura y autónoma -cuando se prometía

15. Texto original en inglés: «Marginal interventions in particular should seem like works of art. People learn to look at their landscapes and the way in which they have developed in a new way. If you can't change the appearance of the landscape in a major way, you can at least influence the way in which people look at it. A marginal approach in terms of landscape and pleasure» (Traducción de Ángel Cordero).

16. Texto original en inglés: «because whatever is sown in the earth typically comes to fruition long after the original gardener has died. This is the meaning. This is the meaning of the metaphor of continuity that Michel Corajoud uses to contrast his theories to the ideologies of domination and wilful imposition: that of a conversation» (Traducción de Ángel Cordero).

Feminismo/s 32, diciembre 2018, pp. 135-155 
una «intrínseca capacidad de la sociedad para identificarse» ${ }^{17}$ (Girot 7) con el paisaje- le ha restado incidencia en la crítica profesional. Esto resulta aún más paradójico dado que su distanciamiento de los focos del star system no resulta de una simple decisión personal, sino que conlleva una actitud resistente a los ritmos de los estudios profesionales, todavía dominados por el frenesí arquitectónico y sus ancestrales connotaciones jerárquicas: ¿acaso no se pretendía que el jardín fuera "parte del grado de entendimiento y respeto que nos gustaría tener por los ritmos naturales» ${ }^{18}$ ? (Girot 7 ). Tal vez esas palabras fueran una simple fantasía finisecular o demasiado avanzadas para un tiempo en que la superproducción y la aceleración del ritmo siguen siendo los caminos de la ortodoxia. Aunque olvidada en exceso, la sosegada obra de Christine Dalnoky seguirá siendo un foco decisivo para el debate sobre el paisaje y, por lo tanto, sobre la ciudad y la cultura contemporáneas.

\section{REFERENCIAS BIBLIOGRÁFICAS}

Alba, Dominique, Patrice Carmouze, Anne Hidalgo, Christine Dalnoky, Jacques Ferrier y François Leclercq. Architecture = Durable. Arsenal TV, 2008. Pavillon de l'Arsenal. 30 de mayo de 2018. <http://www.pavillon-arsenal.com/fr/arsenal-tv/conferences/questions-dactualite/8849-architecture-durable.html>

Beveridge, Charles E. y Paul Rocheleau. Frederic Law Olmstead. Designing the American Landscape. Ed. David Larkin. New York: Universe Publishing, 1998. Carlow, Vanessa Miriam. «The Relevance of Thinking Rural!». Ruralism. Ed. Vanessa Miriam Carlow. Berlin: Jovis Verlag, 2016. 6-9.

Cipriani, Marialuisa y Elena Farnè. «Paesaggi della memoria e dell'innovazione. Ri-abitare i paesaggi della riforma agraria foggiana». Ri-Vista ricerche per la progettazione del paesaggio (julio-diciembre 2012): 126-135.

Corner, James. «Recovering landscapes as a Critical Cultural Practice». Recovering Landscape: Essays in Contemporary Landscape Architecture. Ed. James Corner. New York: Princeton Architectural Press, 1999. 1-26.

Dalnoky, Christine. «Paysage». El Parque del Agua. Zaragoza: Expoagua Zaragoza 2008, 2008. 48-56.

17. Texto original en inglés: «the inherent capacity of society to identify with» (Traducción de Ángel Cordero).

18. Texto original en inglés: «It is not in part the degree of understanding and respect that we would like to have for natural rhythms?» (Traducción de Ángel Cordero).

Feminismo/s 32, diciembre 2018, pp. 135-155 
Dalnoky, Christine y Patrick Solvet. Tous Ego. L’Abecedaire. París: Sens et Tonka Editéurs, 2004.

De la Cal, Pablo. «El Parque del Agua en el sistema de espacios libres de Zaragoza». El Parque del Agua. Zaragoza: Expoagua Zaragoza 2008, 2008. 66-74.

Defrain, François, Olivier Souquet y Christine Dalnoky. «Conversión de las minas de ocre en un espacio turístico. Bruoux. Francia». Paisea, revista de paisajismo 16 (2011): 24-27.

Desvignes, Michel y Christine Dalnoky. «Parcours dans le Paysage des Hauts-deSeine». Topos 13. París: CAUE 92, 1994.

Girot, Christophe: «Foreword». Changes in scenery. Contemporary Landscape architecture in Europe. Ed. Thies Schröder. Basel/Berlin/Boston: Birkhäuser, 2002. 6-9.

Jackson, John Brinckerhoff. Discovering the Vernacular Landscape. New Haven: Yale University Press, 1984.

Jover, Margarita. «Paisaje, materia de pasado y de futuro». El Parque del Agua. Zaragoza: Expoagua Zaragoza 2008, 2008. 12-17.

López, Marcelo y José Luis Garín. «Estructura técnica hidráulica del Parque del Agua de Zaragoza». El Parque del Agua. Zaragoza: Expoagua Zaragoza 2008, 2008. 138-149.

Marot, Sébastien. Suburbanismo y el arte de la memoria. Barcelona: Editorial Gustavo Gili, 2003.

- «The Reclaiming of Sites». Recovering Landscape. Essays in Contemporary Landscape Architecture. Ed. James Corner. New York: Princeton Architectural Press, 1999. 45-57.

Schröder, Thies: Changes in scenery. Contemporary Landscape architecture in Europe. Basel/Berlin/Boston: Birkhäuser, 2002.

Tafuri, Manfredo. Sobre el Renacimiento. Principios, ciudades, arquitectos. Madrid: Ediciones Cátedra, 1995. 